\title{
Frequency of Epidermal Growth Factor Mutation Status and Its Effect on Outcome of Patients with Adenocarcinoma of the Lung
}

\author{
Hanan E. Shafik ${ }^{1,2 * \#, ~ S a l e m ~ H . ~ A l-S h e m a r r i 1 ~}{ }^{1,3 \#, ~ F a h e d ~ A l-E n e z i ~}{ }^{1 \#, ~ M o h a m e d ~ A s h o u r ~}{ }^{1,4 \#,}$ \\ Eman Khalaf ${ }^{1 \#, ~ A h m e d ~ I . ~ A l-K h o d a r y ~}{ }^{1,2 \#}$ \\ ${ }^{1}$ Kuwait Cancer Control Center, Kuwait City, Kuwait \\ ${ }^{2}$ Department of Medical Oncology, National Cancer Institute (NCI), Cairo, Egypt \\ ${ }^{3}$ Department of Medicine, Faculty of Medicine, Kuwait University, Kuwait City, Kuwait \\ ${ }^{4}$ Department of Clinical Oncology, AL-Azhar University, Cairo, Egypt \\ Email: "ezzathanan@yahoo.com, Salem61@gmail.com, falenezi05@yahoo.com, Dr ashour2000@yahoo.com, \\ Emankhalaf22@yahoo.com, A elkhodary2003@yahoo.com
}

Received 25 July 2014; revised 20 August 2014; accepted 14 September 2014

Copyright (C) 2014 by authors and Scientific Research Publishing Inc.

This work is licensed under the Creative Commons Attribution International License (CC BY).

http://creativecommons.org/licenses/by/4.0/

cC) (i) Open Access

\section{Abstract}

The frequency of EGFR mutations is ethnicity-dependent, with a higher proportion in Asian population than in whites. The prevalence of these mutations among Arab patients is unknown. The objective of this study was to report the frequency and spectrum of EGFR mutations in our population with lung adenocarcinoma, and the effect of this mutation on treatment outcome. Tumor specimens from 81 patients histological diagnosed as NSCLC adenocarcinoma were reviewed by our local pathologist and then sent to Lab 21 London, UK. Samples were tested for 28 mutations in EGFR gene. Positive patients received TKI and negative patients received chemotherapy. Data were collected and retrospectively analyzed to determine frequency and spectrum of EGFR positivity. Patients were followed for time to progression (TTP) and overall survival (OS). Overall frequency of EGFR mutation was 37\%; Del 19 (46.6\%), L858R (40\%), D719x and insertion 20 (6.7\%) for each. Females and nonsmokers exhibited a statistically significant higher EGFR positivity $(P=0.003$, 0.059 ) respectively. Overall response rate (ORR) was $76.6 \%$ and $43 \%$ in EGFR positive and negative cases respectively $(P=0.002)$. There was a statistically significant difference in TTP and $0 S$ between EGFR positive and negative patients $(P=0.035$ and 0.039 respectively). Arab patients exhibit an EGFR mutation pattern that is closer to Asian population. EGFR gene mutation subtypes are the same as that reported worldwide. A statistically significant TTP and OS benefit was noticed in EGFR positive patients compared to EGFR negative cases.

\footnotetext{
"Corresponding author.

${ }^{\#}$ All authors have contributed significantly.
}

How to cite this paper: Shafik, H.E., et al. (2014) Frequency of Epidermal Growth Factor Mutation Status and Its Effect on Outcome of Patients with Adenocarcinoma of the Lung. Journal of Cancer Therapy, 5, 1012-1020.

http://dx.doi.org/10.4236/jct.2014.511106 


\section{Keywords}

\section{Adenocarcinoma, EGFR, NSCLC, Outcome}

\section{Introduction}

Worldwide, approximately 1.5 million new cases of lung cancer are diagnosed each year. According to Kuwait Cancer Registry 2010, the incidence of lung cancer is 12.9 cases/100,000 persons every year; about half of the patients are adenocarcinoma [1]. Platinum-based chemotherapy is considered standard of care worldwide for patients with advanced NSCLC. Approximately one third of patients obtain an objective response with first-line chemotherapy, and another 20\% - 30\% achieves temporary disease stabilization. Five-year survival rate across all stages of the disease is approximately $14 \%$ [2].

Patients who are never smokers, females, adenocarcinomas, and Asians have a relatively high incidence of somatic mutations in the region of the EGFR gene that encodes the tyrosine kinase domain [3]. Mutations in the EGFR tyrosine kinase are observed in approximately 15\% of NSCLC adenocarcinoma in the United States and occur more frequently in nonsmokers. In Asian populations, the incidence of EGFR mutations is substantially higher, reaching up to $62 \%$ [4].

The commonest mutation is exon 19 deletion (45\%) followed by exon 20 (L858R) (40\%). Other mutations like exon 21 (L861Q) and exon 18 (G719X) are less common [3]. T790 mutation is associated with acquired resistance to Tyrosine Kinase Inhibitors (TKI) therapy [5]. Studies have shown that patients with pulmonary adenocarcinoma who had a base-pair deletion at exon 19 (del746-A750) or a point mutation at exon 21 (L858R) were highly responsive to EGFR TKI [6]. The overall response rate (ORR) to erlotinib among patients with EGFR gene mutations (exon 19 deletion or exon 21 L858R mutations) was almost 4 times more than that among patients with wild-type EGFR (27\% vs. 7\%; P = 0.03) [7].

Data from 13 phase III trials in which an EGFR TKI was compared with platinum-based chemotherapy were summarized in a meta-analysis that included data of 2620 patients (1475 EGFR mutation positive and 1145 mutation negative). Time to progression (TTP) was significantly prolonged in EGFR TKI group (Hazard ratio (HR) 0.43 , 95\% CI 0.38 - 0.49), while no effect on OS was observed (HR 1.01, 95\% CI 0.87 - 1.18) [8].

Gefitinib was compared with cytotoxic chemotherapy as the initial therapy in three randomized trials in patients with advanced NSCLC whose tumors contained activating EGFR mutations. In the IPASS trial for the $60 \%$ of patients whose tumors contained an EGFR mutation, progression free survival (PFS) was significantly prolonged with gefitinib compared with carboplatin plus paclitaxel (median 9.5 versus 6.3 months, HR 0.48 (95\% CI 0.36 0.64) [6]. OS was not increased (median 22 months in both groups, HR 1.00 (95\% CI 0.79 - 1.02) [9]. Two additional phase III trials were conducted exclusively in patients with EGFR mutations; the North-East Japan Study Group 002 trial [10] [11] and the West Japan Oncology Group 172 trial [12]. The overall results and magnitude of the benefit were essentially similar to the IPASS trial. Erlotinib was compared to chemotherapy in two randomized trials in patients all of whose tumors with activated EGFR mutation. In the OPTIMAL trial from China, 154 patients were randomly assigned to erlotinib or gemcitabine plus carboplatin [13]. Treatment with erlotinib significantly improved PFS compared with chemotherapy (13.1 versus 4.6 months, HR 0.16, 95\% CI 0.10 - 0.26). In the EURTAC trial, 174 patients were randomly assigned to erlotinib or a platinum-based chemotherapy doublet [14]. PFS, the primary endpoint of the trial, was significantly increased with erlotinib compared with chemotherapy (median 9.7 versus 5.2 months, HR 0.37 , 95\% CI 0.25 - 0.54). There was no significant difference in median OS (19.3 and 19.5 months, respectively).

Our study objective is to determine the percentage of patients who carry a sensitizing EGFR mutation and effect of this mutation on their treatment outcome.

\section{Patients and Methods}

Between June 2010 and February 2013, 81 patients received at Kuwait Cancer Control Center (KCCC) Outpatient Clinic were diagnosed with NSCLC (adenocarcinoma). Core tissue biopsies from those patients were reviewed by local pathologists, and then samples were sent to Lab 21 London, UK to be tested for EGFR mutation using 
Therascreen EGFR PCR kit. Twenty-eight different mutations in EGFR gene were studied with 3 targets being mutation specific (L858R, L861Q, S7681) the other 25 targets were detected if present but were grouped into 3 types (deletion 19, G719x and insertion 20). EGFR mutation test result was reported as exhibiting or not sensitizing mutation to EGFR TKI based therapy based on the review by Sharma et al. [15].

As per the local clinical practice guidelines, patients carrying one of the sensitizing EGFR mutations received TKI while patients lacking sensitizing mutations received chemotherapy. Three patients in the EGFR negative group and two in EGFR positive group were not evaluable for response. Patients receiving TKI were reevaluated for response every 3 months while those receiving chemotherapy were reevaluated after third and sixth cycles.

Data of this group of patients were then retrospectively collected and analyzed to find out percentage of EGFR positivity, the effect of this mutation on their response to standard treatment as well as on TTP and OS. Response was evaluated using RECIST criteria.

\section{Statistical Methods}

Data management and analysis was performed using SPSS, version 14. Categorical data were summarized as percentages, numerical data were summarized using means and standard deviation or medians and range. Hazard ratios were calculated using cox regression. OS was defined as the time from diagnosis to the time of death from any cause. Patients who were alive on the date of last follow-up were censored on that date. TTP was defined as the time from starting therapy until documented progression. For patients without DP at the time of analysis, the date of last follow-up was considered right-censored. OS and TTP were estimated using the KaplanMeier analysis.

\section{Results}

A total of 81 patients diagnosed with NSCLC adenocarcinoma subtype were studied for EGFR mutation which was detected in 30 patients (37\%). Exon 19 deletion was detected in 14 patients (46.6\%), exon 20 (L858R) mutation in 12 patients (40\%), D719x in 2 patients (6.7\%), insertion 20 in 2 patients (6.7\%). None of the patients exhibited S7681 mutation. The mean age of all patients was $61.8 \pm 10.23$. Males represented $64.2 \%$, and smokers represented $28.4 \%$ of the patients. Approximately $57 \%$ were Kuwaitis and $43.2 \%$ were from other Arab countries. Performance status of $88 \%$ of the patients was 0 to II. Patients with stage IV at diagnosis represented $89 \%$ and the rest were stage IIIB (Table 1 ).

EGFR mutation was detected in 17 females (58.6\%) and 13 males (25\%) $(\mathrm{P}=0.003)$. Approximately $43 \%$ of nonsmokers and $22 \%$ of smokers exhibited EGFR mutation $(\mathrm{P}=0.059)$. Females exhibited a statistically significant higher EGFR positivity compared to males, odds ratio (OR) of 4.06, nonsmokers had higher incidence of EGFR positivity compared to smokers OR of 0.56 (Table 2).

Patients proved to carry one of the sensitive EGFR mutations received a TKI as first line, while patients lacking sensitive mutation received chemotherapy. Patients' characteristics' were balanced between the two groups (Table 2). In the TKI group 20 patients (66.7\%) received Erlotinib, 10 patients (33.3\%) received Gefetinib. Mean duration of treatment was 8.8 months (95\% CI 6.3 - 11.4). Patient who proved to lack sensitive mutations received chemotherapy; 30 patients (58.8\%) received carboplatin/paclitaxel, 18 patients (35.2\%) received cisplatin/pemetrexed, and 3 patients received carboplatin single agent. Mean number of chemotherapy cycles was 4 (95\% CI 3.7 - 4.6). 19 (67.9\%) of EGFR positive patients achieved PR, 4 (14.3\%) achieved SD, and 5 (17.9\%) progressed; while 15 (31.2\%) of EGFR negative patients experienced PR, 7 (14.6\%) experienced SD, and 26 (54.2\%) progressed. Partial response (PR) plus stable disease (SD) was 76.6\% and $43 \%$ in EGFR positive and negative cases respectively a difference which was statistically significant $\mathrm{P}=0.002$.

Approximately $65 \%$ of patients receiving TKI experienced skin rash, 52\% experienced fatigue, 37\% experienced diarrhea and 6\% experienced conjunctivitis. Approximately 33\% of patients receiving chemotherapy developed Grade IV neutropenia and was complicated by fever in $4 \%$ of the patients, $14 \%$ of patients developed Grade III neuropathy and about $18 \%$ experienced nausea and vomiting.

The median follow up duration was $15.4 \pm 13.7$ months. TTP in EGFR positive patients was 13.58 (95\% CI 10.6 - 16.556), compared to 6.74 months in EGFR negative cases (95\% CI 3.28 - 10.2), a difference which is statistically significant, $\mathrm{P}=0.035$, HR for progression is 0.579 (Figure 1). OS was 21.8 months (95\% CI 15.6 - 27.9) 
in EGFR positive compared to 9.9 months (95\% CI 7.628 - 12.17) in EGFR negative cases, a difference which was statistically significant $P=0.039$. HR for death was 0.514 (Figure 2). No statistically significant difference in median OS was noticed between males and females or smokers and nonsmokers $(\mathrm{P}=0.356$ and 0.949$)$ respectively (Figure 3 and Figure 4).

Table 1. Patient's characteristics.

\begin{tabular}{|c|c|c|}
\hline \multirow[t]{2}{*}{ Patients Characteristics } & \multicolumn{2}{|c|}{ Frequencies } \\
\hline & $\mathrm{n}$ & $\%$ \\
\hline Mean age & 61.8 & \pm 10.2 \\
\hline \multicolumn{3}{|l|}{ Age groups } \\
\hline$\leq 60$ & 34 & 42 \\
\hline$>60$ & 47 & 58 \\
\hline \multicolumn{3}{|l|}{ Sex } \\
\hline Male & 52 & 64.2 \\
\hline Female & 29 & 35.3 \\
\hline \multicolumn{3}{|l|}{ Smoking } \\
\hline Smoker & 23 & 28.4 \\
\hline Nonsmoker & 58 & 71.6 \\
\hline \multicolumn{3}{|l|}{ Nationality } \\
\hline Kuwaiti & 46 & 56.8 \\
\hline Non-Kuwaiti & 35 & 43.2 \\
\hline \multicolumn{3}{|l|}{$* \mathrm{PS}$} \\
\hline 0 & 18 & 22 \\
\hline 1 & 28 & 34.6 \\
\hline 2 & 25 & 31 \\
\hline 3 & 10 & 12.4 \\
\hline 4 & 0 & 0 \\
\hline \multicolumn{3}{|l|}{ Stage } \\
\hline III-B & 9 & 11 \\
\hline IV & 72 & 89 \\
\hline \multicolumn{3}{|l|}{ *EGFR } \\
\hline *EGFR positive & 30 & 37 \\
\hline *EGFR negative & 51 & 63 \\
\hline \multicolumn{3}{|l|}{ *EGFR mutation type } \\
\hline Deletion 19 & 14 & 46.6 \\
\hline L858R & 12 & 40 \\
\hline L861Q & 2 & 6.7 \\
\hline G719X & 2 & 6.7 \\
\hline
\end{tabular}

PS: Performance status; EGFR: Epidermal growth factor receptor. 
Table 2. Clinicopathological characteristics in relation to EGFR mutation status.

\begin{tabular}{|c|c|c|c|c|c|}
\hline \multirow[t]{2}{*}{ Patients Characteristics } & \multicolumn{2}{|c|}{ EGRF Negative } & \multicolumn{2}{|c|}{ EGFR Positive } & \multirow[t]{2}{*}{ P-Value } \\
\hline & $\mathrm{n}$ & $\%$ & $\mathrm{n}$ & $\%$ & \\
\hline \multicolumn{6}{|l|}{ Age groups } \\
\hline$\leq 60$ & 22 & 64.7 & 12 & 35.4 & 0.48 \\
\hline$>60$ & 29 & 61.7 & 18 & 38.3 & \\
\hline \multicolumn{6}{|l|}{ sex } \\
\hline Male & 39 & 75 & 13 & 25 & 0.01 \\
\hline Female & 12 & 41.4 & 17 & 58.6 & \\
\hline Nationality & & & & & 0.12 \\
\hline Kuwaiti & 32 & 69.6 & 14 & 30.4 & \\
\hline Non-Kuwaiti & 19 & 54.3 & 16 & 45.7 & \\
\hline smoking & & & & & 0.06 \\
\hline Smoker & 18 & 78.3 & 5 & 21.7 & \\
\hline Nonsmoker & 33 & 56.9 & 25 & 43.1 & \\
\hline Stage & & & & & 0.65 \\
\hline III-B & 5 & 55.6 & 4 & 44.4 & \\
\hline IV & 46 & 63.9 & 26 & 36.1 & \\
\hline *PS & & & & & 0.34 \\
\hline 0 & 11 & 61.1 & 7 & 38.9 & \\
\hline 1 & 14 & 50 & 14 & 50 & \\
\hline 2 & 19 & 76 & 6 & 34 & \\
\hline 3 & 4 & 80 & 1 & 20 & \\
\hline 4 & 3 & 60 & 2 & 40 & \\
\hline \multicolumn{6}{|l|}{ Response } \\
\hline$* \mathrm{PR}+\mathrm{SD}$ & 22 & 48.9 & 23 & 51.1 & 0.01 \\
\hline$* \mathrm{DP}$ & 26 & 80 & 5 & 20 & \\
\hline
\end{tabular}

PS: Performance status; PR: Partial response; SD: Stable disease; DP: Disease progression.

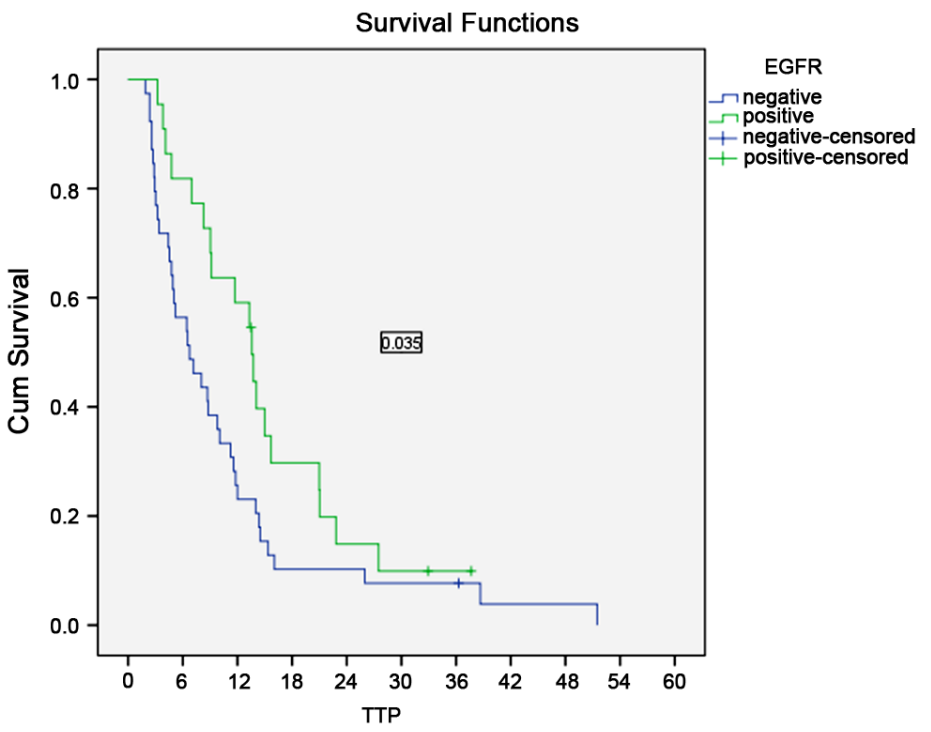

Figure 1. Effect of EGFR status on time to progression. 
Title

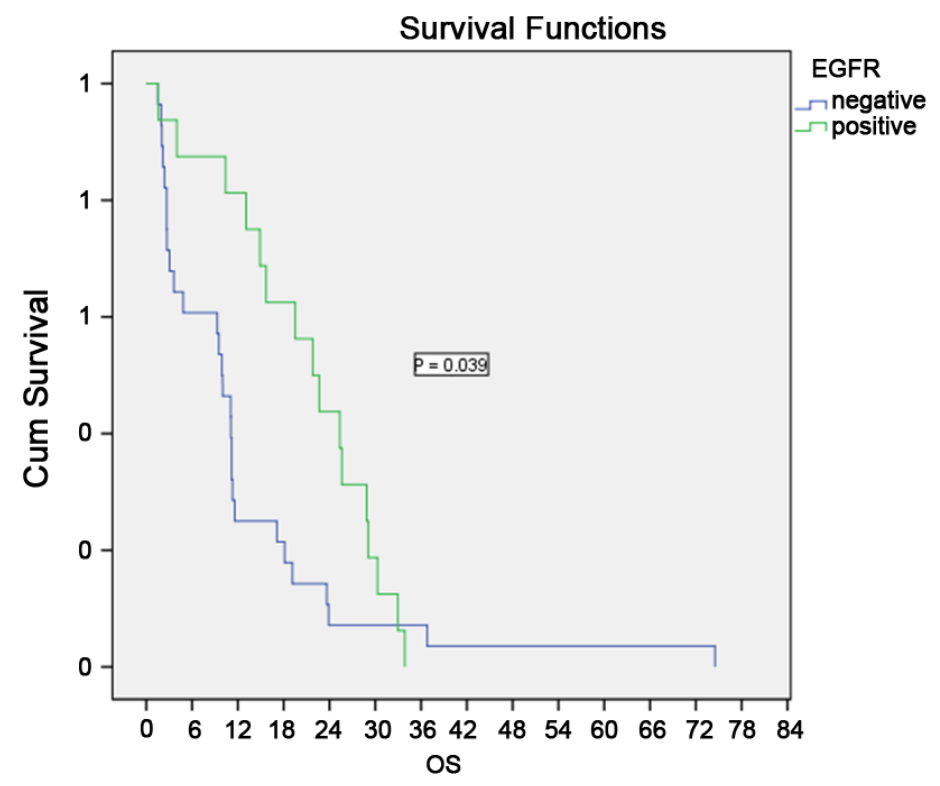

Figure 2. Effect of EGFR status on overall survival.

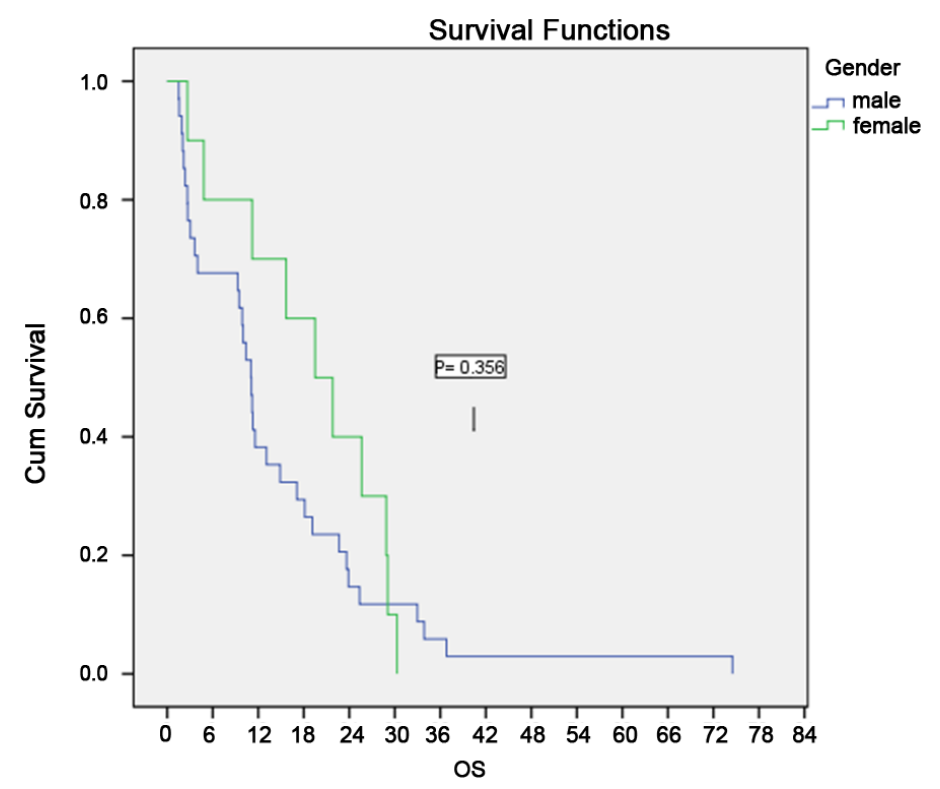

Figure 3. Effect of gender on overall survival.

\section{Discussion}

EGFR mutation was detected in 37\% of our patients. This percentage was higher than that detected in Americans (15\%) and lower than that detected by the PIONEER study in Asians. Incidence of EGFR mutation in Asians was higher in females than males ( $61.1 \%$ and $44 \%$ respectively), and in never smokers than patients smoking $>50$ pack-year (60.7\% and 31.4\% respectively), these findings were consistent with our data [4].

Exon 19 Del was detected in 46.6\%, exon 21 (L858R) mutations in 40\%, D719x and insertion 20 in 6.7\%. None of our patients exhibited S7681 mutation. These findings are consistent with those reported by Sharma et al. where exon 19 Del was detected in 45\% of patients, L858R mutation in 40\% - 45\% of patients, D719x in 5\% of patients, insertion 20 in less than $1 \%$ of patient [15]. Our data were consistent with that reported in IPASS trial where 96\% of patients exhibited either an exon 19 deletion or the exon 21 L858R mutations [9]. 


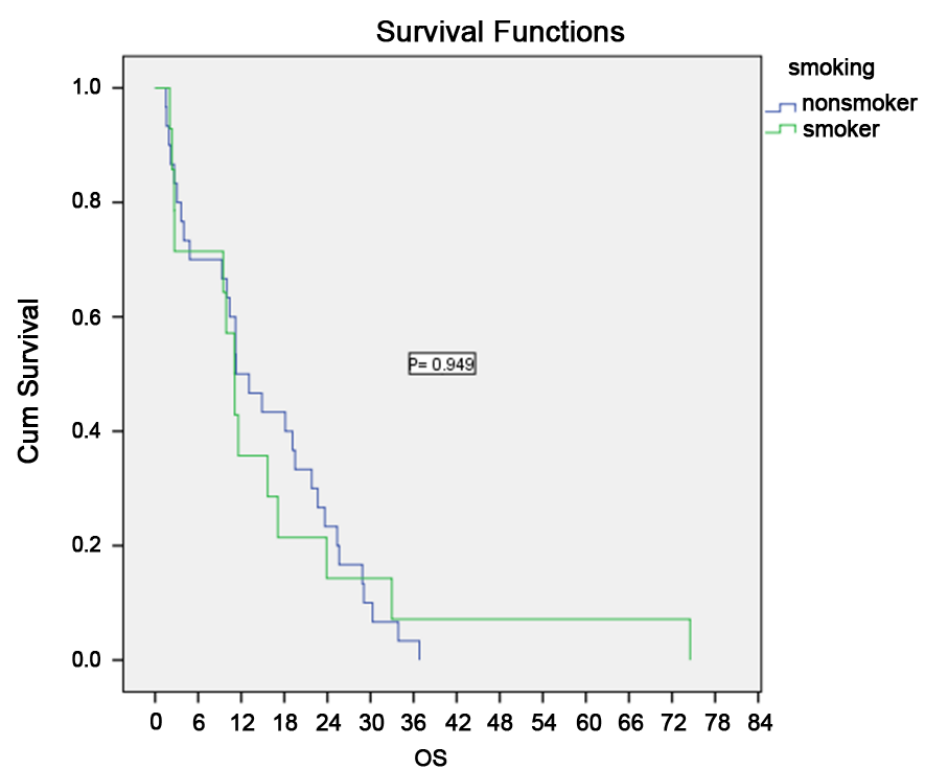

Figure 4. Effect of smoking on overall survival.

A statistically significant difference was noticed in RRs between patients receiving TKI and those receiving combination chemotherapy in our patients population (76.6\%, 43\% respectively) $\mathrm{P}=0.002$. These data are consistent with those reported by Mok et al. in IPASS trial, where RRs were $71.2 \%$ vs. $47.3 \%$ for gefitinib and carboplatin plus paclitaxel respectively. In the subgroup of patients who proved to have positive mutation, RRs were $71 \%$ vs. $47 \%$ for gefitinib and carboplatin plus paclitaxel respectively while in EGFR negative patients RRs were $1.1 \%$ vs. $23.5 \%$ favoring chemotherapy [6] [9]. Our RR in EGFR positive group was nearly equivalent to the corresponding group in IPASS trial and slightly higher than corresponding group in EGFR negative patients.

The North-East Japan Study Group 002 trial reported RRs of $(73.7 \%$ and 30.7\%, P < 0.001) for Gefitinib vs. combination chemotherapy [10]. In The OPTIMAL and EURTAC trial, patients with proved EGFR positive mutation were randomized to erlotinib or platinum based chemotherapy. RRs were ( $83 \%$ vs. $36 \%$ ) and ( $58 \%$ vs. $15 \%$ ) respectively. Our RRs for patients with EGFR negative mutation who received chemotherapy were less than that reported previously in clinical trials. This may be attributed to the fact that all patients in those three trial exhibit EGFR mutation, and the response as well as survival for EGFR positive patients is better than that for EGFR negative [13] [14].

TTP in our EGFR positive patients group was 13.58 (95\% CI 10.6 - 16.556), compared to 6.74 months in EGFR negative cases ( $95 \%$ CI 3.28 - 10.2), a difference which was statistically significant, $P=0.035$, HR for progression was 0.579 . These data were consistent with those reported by Moketal, where TTP was significantly better with gefitinib compared to chemotherapy (12-month progression-free rate 25 vs. 7 percent, HR for progression 0.74). Mutations were detected in $60 \%$ of patients for whom TTP was significantly prolonged with gefitinib compared to carboplatin/paclitaxel (median 9.5 versus 6.3 months, HR for progression 0.48) [9].

The West Japan Oncology Group 172 trial showed median TTP of 9.2 months (95\% CI 8.0 - 13.9) versus 6.3 months (95\% CI 5.8 - 7.8; HR 0.489, 95\% CI 0.336 - 0.710, P < 0.0001) [12] and the North-East Japan Study Group 002 trial show nearly the same results median TTP (10.8 months, vs. 5.4 months in the chemotherapy group; hazard ratio, 0.30; (95\% CI, 0.22 to $0.41, \mathrm{P}<0.001)$, as well as a higher ORR $(73.7 \%$ vs. $30.7 \%, \mathrm{P}<0.001)$. The median OS was 30.5 months in the gefitinib group and 23.6 months in the chemotherapy group $P=0.31$, findings that are higher than our findings [10] [11].

In the OPTIMAL trial from China and EURTAC trial from Europe patients with sensitizing mutation were randomly assigned to erlotinib or gemcitabine plus platinum-based chemotherapy doublet. Treatment with erlotinib significantly improved median TTP compared with chemotherapy (13.1 vs. 4.6 months HR 0.16 for progression, 95\% CI 0.10 - 0.26) and (9.7 vs. 5.2 months, HR for progression 0.37, 95\% CI 0.25 - 0.54) respectively [13] [14].

Our data showed an OS of 21.8 months (95\% CI 15.6 - 27.9) compared to 9.9 months (95\% CI 7.63 - 12.17) in EGFR positive and negative cases respectively, a difference which was statistically significant $P=0.039$. HR for 
death was 0.514. The IPASS, OPTIMAL and EURTAC trials all showed no difference in survival between the two groups with a median OS of about 22 months [9] [13] [14]. This OS difference in EGFR and chemotherapy groups in our trial compared to other trials could be attributed to the fact that cross over was allowed in those trials, but not in ours. Moreover, chemotherapy in our trial was given to EGFR negative patients only who are known to be less responsive to treatment than EGFR positive patients.

However, our survival in chemotherapy arm was consistent with survival data reported in chemotherapy trials. A meta-analysis that included individual patient data from 2968 patients in nine trials, comparing cisplatin based to carboplatin based chemotherapy showed median survival of (median 8.4 vs. 9.1 months, HR for death 1.07, 95\% CI 0.99 - 1.15) [16].

Our data were also consistent with TORCH trial [17], where 760 patients were randomly assigned to either firstline erlotinib followed by chemotherapy (cisplatin plus gemcitabine) upon progression or the same first-line chemotherapy followed by erlotinib upon progression. EGFR mutation status was analyzed in 64 percent of cases; among these, 86 percent were EGFR wild type. OS was significantly longer in unselected patients assigned to initial chemotherapy followed by second-line erlotinib (median 11.6 versus 8.7 months, hazard ratio [HR] 1.24, 95\% CI 1.04 - 1.47). For patients known to be EGFR mutation negative, overall survival was significantly longer with initial chemotherapy (median 9.6 versus 6.5 months).

In a phase III trial cisplatin/pemetrexed was compared to cisplatin/gemcitabine as initial therapy. OS in the 847 patients with adenocarcinoma was significantly prolonged with cisplatin plus pemetrexed compared to cisplatin plus gemcitabine (median 12.6 versus 10.9 months). Conversely, cisplatin plus gemcitabine was superior to cisplatin plus pemetrexed in the 473 patients with squamous cell carcinoma (median 10.8 versus 9.4 months) [18] [19].

\section{Conclusion}

Our patients exhibit an EGFR mutation pattern that is closer to Asian population. EGFR gene mutation subtypes are the same as that reported worldwide. A statistically significant TTP and OS benefit was noticed in EGFR positive patients compared to EGFR negative cases.

\section{Conflict of Interest}

No conflicts of interest are declared.

\section{References}

[1] Elbasmi, A., Al-Asfour, A., Al-Nesf, Y. and Al-Awadi, A. (2010) Cancer in Kuwait Magnitude of the Problem. GJO, 8 , 7-14.

[2] Azzoli, C.G., Baker, S.J., Temin, S., et al. (2009) American Society of Clinical Oncology Clinical Practice Guideline Update on Chemotherapy for Stage IV Non-Small-Cell Lung Cancer. Journal of Clinical Oncology, 27, 6251-6266. http://dx.doi.org/10.1200/JCO.2009.23.5622

[3] Shigematsu, H., Lin, L., Takahashi, T., et al. (2005) Clinical and Biological Features Associated with Epidermal Growth Factor Receptor Gene Mutations in Lung Cancers. Journal of the National Cancer Institute, 97, 339-346. http://dx.doi.org/10.1093/jnci/dji055

[4] Shi, Y., Au, J.S., Thongprasert, S., et al. (2014) A Prospective, Molecular Epidemiology Study of EGFR Mutations in Asian Patients with Advanced Non-Small-Cell Lung Cancer of Adenocarcinoma Histology (PIONEER). Journal of Thoracic Oncology, 9, 154-162. http://dx.doi.org/10.1097/JTO.0000000000000033

[5] Reily, G.J., Politi, K.A., Miller, V.A. and Pao, W. (2006) Update on Epidermal Growth Factor Receptor Mutation in NSCLC. Clinical Cancer Research, 12, 7232-7241. http://dx.doi.org/10.1158/1078-0432.CCR-06-0658

[6] Pao, W., Miller, V., Zakowski, M., et al. (2004) EGF Receptor Gene Mutations Are Common in Lung Cancers from "Never Smokers" and Are Associated with Sensitivity of Tumors to Gefitinib and Erlotinib. The Proceedings of the National Academy of Sciences of the United States of America, 101, 13306-13311. http://dx.doi.org/10.1073/pnas.0405220101

[7] Miller, V.A., Riely, G.J., Zakowski, M.F., et al. (2008) Molecular Characteristics of Broncho-Alvealar and Adenocarcinoma, Broncho-Alveolar Carcinoma Subtype, Predict Response to Erlotinib. Journal of Clinical Oncology, 26, 1472-1478. http://dx.doi.org/10.1200/JCO.2007.13.0062

[8] Lee, C.K., Brown, C., Gralla, R.J., et al. (2013) Impact of EGFR Inhibitor in Non-Small Cell Lung Cancer on Progres- 
sion-Free and Overall Survival: A Meta-Analysis. Journal of the National Cancer Institute, 105, 595-605. http://dx.doi.org/10.1093/jnci/djt072

[9] Fukuoka, M., Wu, Y.L., Thongprasert, S., et al. (2011) Biomarker Analyses and Final Overall Survival Results from a Phase III, Randomized, Open-Label, First-Line Study of Gefitinib versus Carboplatin/Paclitaxel in Clinically Selected Patients with Advanced Non-Small-Cell Lung Cancer in Asia (IPASS). Journal of Clinical Oncology, 29, $2866-2874$. http://dx.doi.org/10.1200/JCO.2010.33.4235

[10] Inoue, A., Kobayashi, K., Maemondo, M., et al. (2013) Updated Overall Survival Results from a Randomized Phase III Trial Comparing Gefitinib with Carboplatin-Paclitaxel for Chemo-Naïve Non-Small Cell Lung Cancer with Sensitive EGFR Gene Mutations (NEJ002). Annals of Oncology, 24, 54-59. http://dx.doi.org/10.1093/annonc/mds214

[11] Maemondo, M., Inoue, A., Kobayashi, K., et al. (2010) Gefitinib or Chemotherapy for Non-Small-Cell Lung Cancer with Mutated EGFR. The New England Journal of Medicine, 362, 2380-2388. http://dx.doi.org/10.1056/NEJMoa0909530

[12] Mitsudomi, T., Morita, S., Yatabe, Y., et al. (2010) Gefitinib versus Cisplatin plus Docetaxel in Patients with NonSmall-Cell Lung Cancer Harboring Mutations of the Epidermal Growth Factor Receptor (WJTOG3405): An Open Label, Randomised Phase 3 Trial. The Lancet Oncology, 11, 121-128. http://dx.doi.org/10.1016/S1470-2045(09)70364-X

[13] Zhou, C., Wu, Y.L., Chen, G., et al. (2011) Erlotinib versus Chemotherapy as First-Line Treatment for Patients with Advanced EGFR Mutation-Positive Non-Small-Cell Lung Cancer (OPTIMAL, CTONG-0802): A Multicentre, OpenLabel, Randomised, Phase 3 Study. The Lancet Oncology, 12, 735-742. http://dx.doi.org/10.1016/S1470-2045(11)70184-X

[14] Rosell, R., Carcereny, E., Gervais, R., et al. (2012) Erlotinib versus Standard Chemotherapy as First-Line Treatment for European Patients with Advanced EGFR Mutation-Positive Non-Small-Cell Lung Cancer (EURTAC): A Multicentre, Open-Label, Randomised Phase 3 Trial. The Lancet Oncology, 13, 239-246. http://dx.doi.org/10.1016/S1470-2045(11)70393-X

[15] Sharma, S.V., Bell, D.W., Settleman, J. and Haber, D.A. (2007) Epidermal Growth Factor Mutation in Lung Cancer. Nature Reviews Cancer, 7, 169-181. http://dx.doi.org/10.1038/nrc2088

[16] Johnson, B., et al. (2013) A Multicenter Effort to Identify Driver Mutations and Employ Targeted Therapy in Patients with Lung Adenocarcinomas: The Lung Cancer Mutation Consortium. JCO, 31, Abstract 8019.

[17] Ardizzoni, A., Boni, L., Tiseo, M., et al. (2007) Cisplatin- versus Carboplatin-Based Chemotherapy in First-Line Treatment of Advanced Non-Small-Cell Lung Cancer: An Individual Patient Data Meta-Analysis. Journal of the National Cancer Institute, 99, 847-857. http://dx.doi.org/10.1093/jnci/djk196

[18] Scagliotti, G.V., Parikh, P., von Pawel, J., et al. (2008) Phase III Study Comparing Cisplatin plus Gemcitabine with Cisplatin plus Pemetrexed in Chemotherapy-Naive Patients with Advanced-Stage Non-Small-Cell Lung Cancer. Journal of Clinical Oncology, 26, 3543-3551. http://dx.doi.org/10.1200/JCO.2007.15.0375

[19] Syrigos, K.N., Vansteenkiste, J., Parikh, P., von Pawel, J., Manegold, C., Martins, R.G., et al. (2010) Prognostic and Predictive Factors in a Randomized Phase III Trial Comparing Cisplatin-Pemetrexed versus Cisplatin-Gemcitabine in Advanced Non-Small-Cell Lung Cancer. Annals of Oncology, 21, 556-561. http://dx.doi.org/10.1093/annonc/mdp392 
Scientific Research Publishing (SCIRP) is one of the largest Open Access journal publishers. It is currently publishing more than 200 open access, online, peer-reviewed journals covering a wide range of academic disciplines. SCIRP serves the worldwide academic communities and contributes to the progress and application of science with its publication.

Other selected journals from SCIRP are listed as below. Submit your manuscript to us via either submit@scirp.org or Online Submission Portal.
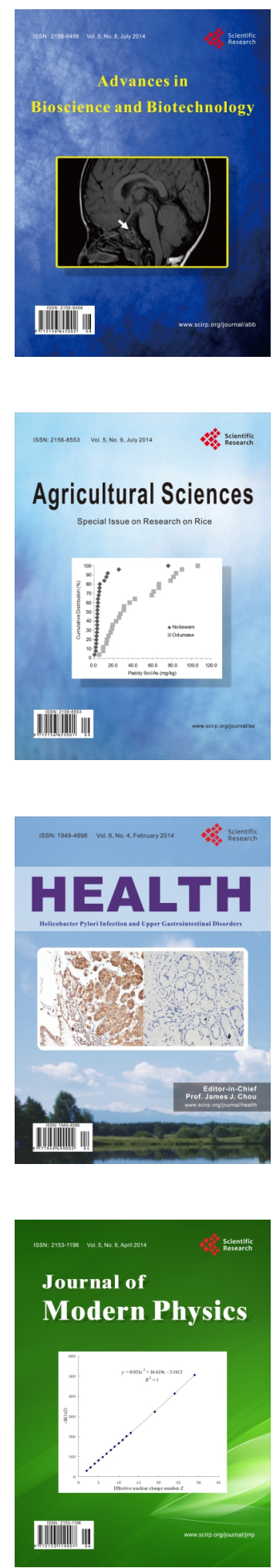
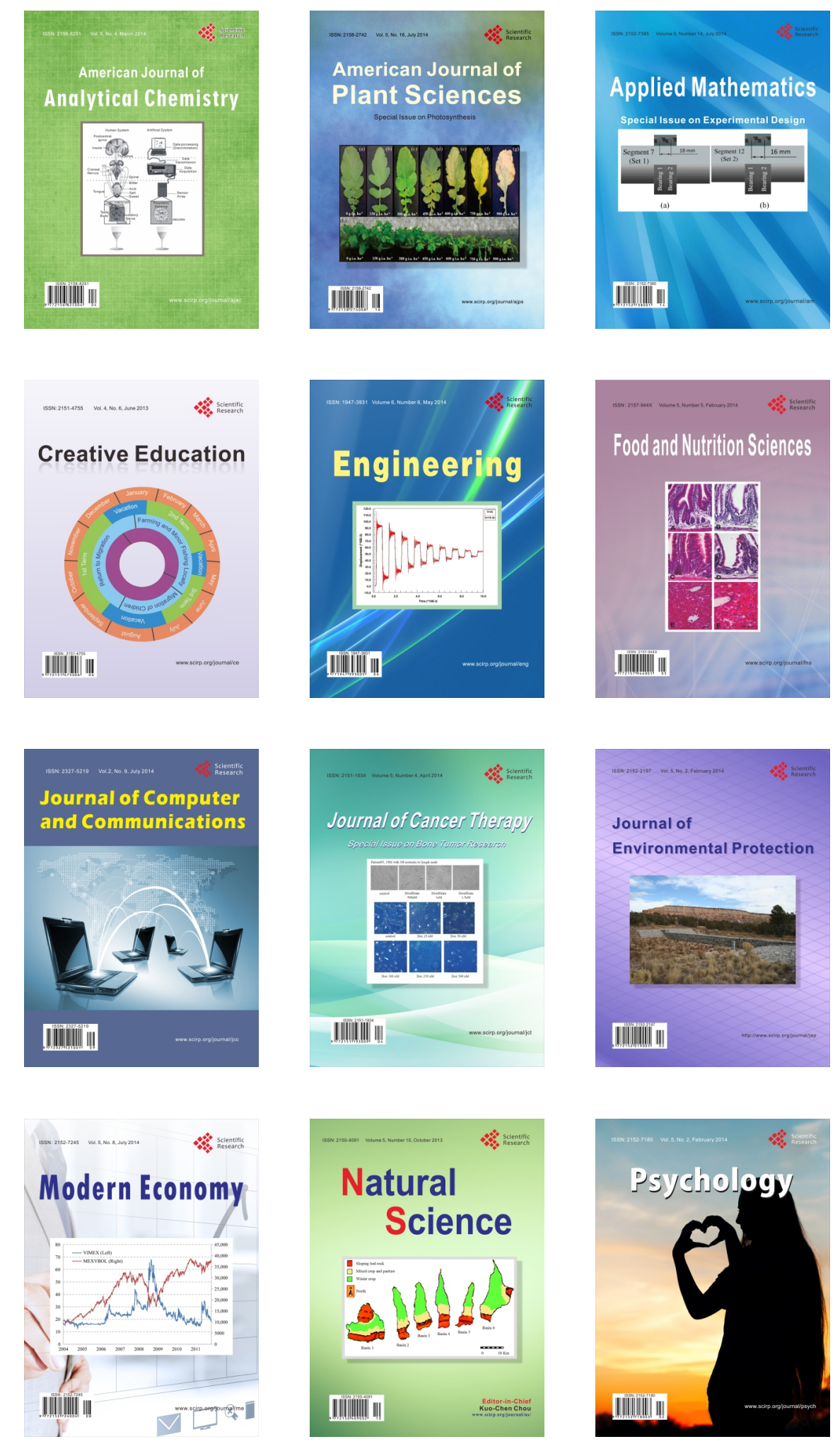